This item was submitted to Loughborough's Research Repository by the author.

Items in Figshare are protected by copyright, with all rights reserved, unless otherwise indicated.

\title{
Base pressure and flow-field measurements on a generic SUV model
}

PLEASE CITE THE PUBLISHED VERSION

http://dx.doi.org/10.4271/2015-01-1546

\section{PUBLISHER}

(c) SAE International

\section{VERSION}

AM (Accepted Manuscript)

\section{LICENCE}

CC BY-NC-ND 4.0

\section{REPOSITORY RECORD}

Wood, Andrew, Martin A. Passmore, David C. Forbes, Daniel Wood, and Adrian P. Gaylard. 2015. "Base Pressure and Flow-field Measurements on a Generic SUV Model". figshare. https://hdl.handle.net/2134/17659. 


\title{
Base Pressure and Flow-Field Measurements on a Generic SUV Model
}

\author{
Andrew Wood, Martin Passmore, David Forbes, and Daniel Wood Loughborough Univ \\ Adrian Gaylard Jaguar Land Rover (Do NOT enter this information. It will be pulled from participant tab in \\ MyTechZone) \\ (Do NOT enter this information. It will be pulled from participant tab in MyTechZone)
}

\begin{abstract}
The pressure on the base of a vehicle is a major contributor to the aerodynamic drag of all practical vehicle geometries, and for some vehicles, such as an SUV, it is particularly important because it can account for up to $50 \%$ of the overall drag. Understanding the mechanisms that influence the base pressure and developing our simulation tools to ensure that base pressure is accurately predicted are essential requirements for the vehicle design and engineering process.
\end{abstract}

This paper reports an experimental study to investigate the base pressure on a specifically designed generic SUV model. The results from $1 / 4$ scale wind tunnel tests include force and moment data, surface pressures over the base region and particle image velocimetry (PIV) in the wake.

Results are presented for the vehicle in different ride height, underfloor roughness and wheel configurations and the paper includes some description of the experimental errors. Some initial CFD simulations are also reported.

\section{Introduction}

The automotive industry faces a number of challenges in the design and optimization of vehicles for aerodynamic purposes, particularly with the pressing need to reduce energy consumption. However, a number of aspects of vehicle aerodynamics are not sufficiently well understood for further progress in drag reduction and accurate simulation during the design and engineering process. One such area is the numerical prediction of base pressure, where the contribution to the overall drag is very important but the accurate prediction is difficult because of an incomplete understanding of the main physics and flow-field mechanisms. In the case of SUV's the base pressure is a particularly large contribution to the total drag so an understanding of the driving mechanisms is critical. This paper serves a number of complimentary purposes. Firstly a simple generic SUV model is developed and described and made available for ongoing CFD development; secondly a description of the effect of wake structure upon base pressure and the resulting effect on drag is given for this generic geometry. Finally the experimental data will be available for download from the Loughborough University Institutional Repository [1]. The data includes details of the geometry and tunnel setup, full descriptions of data and its location in the model frame of reference.

\section{Methods and Materials}

\section{Model Design}

The use of simplified vehicle models for experimental and computational research is well established in the aerodynamics community and has a proven record in enhancing our understanding of the underlying fundamentals and in the development of CFD methods. Well known examples of this are the Ahmed geometry [2], designed to capture the influence of a specific vehicle feature (the rear slant) or the SAE reference model [3], designed as a general purpose simplified vehicle. The focus of these models has been towards the conventional vehicle configurations, such as fastback, notchback and square-back but none exist to capture the underlying geometry and shape of an SUV. A new model was therefore developed based on an analysis of the large SUV market segment. Twenty-seven external dimensions where taken for a range of 39 vehicles from 12 manufacturers with model years spanning 1970 to 2011. The side profiles are shown overlaid in Figure 1. With normalized measurements and the data color coded with model year.

To define the wind tunnel model the characteristic data was summarized to identify trends and an initial geometry based on these trends generated. This was then further simplified to remove the influence of the large wheel arch eyebrows, often evident in these vehicles, and to remove the tumblehome and backlight rake from the glass house. These simplifications reduce the Reynolds sensitivity of the model and allow a wider range of future parametric studies to be undertaken based on the generic SUV. Finally a radius was applied to all leading edges to prevent local separations that would not be representative of full scale. The size of the radius was based on the work of Newnham [4]. 


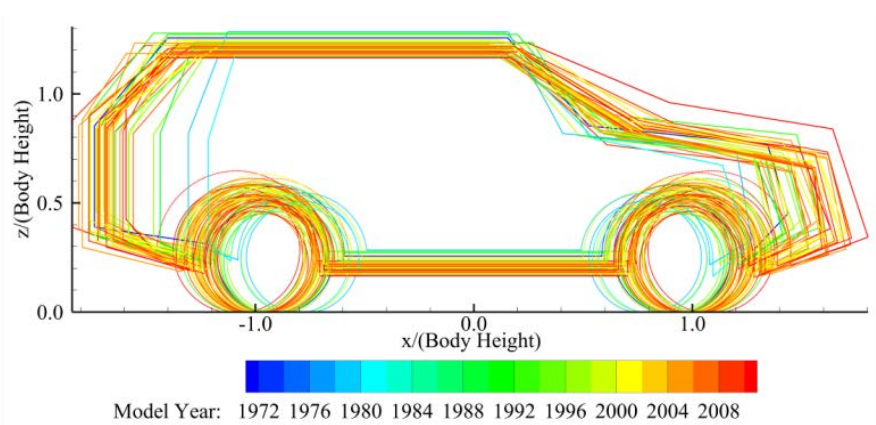

Figure 1 - Outlines of benchmarked SUV models, coloured by model year

The model was designed to facilitate changes in ride height (tunnel floor to model underside), underfloor roughness and for testing with and without wheels. The configurations tested are listed in Table 1, with images of the changes in ride height, underfloor roughness and wheels shown in Figure 2, Figure 3 and Figure 4 respectively. The wheels and tires do not include any detail. The underside of the wheel is flattened to simulate the contact zone and there is a small gap $(2 \mathrm{~mm})$ between the underside of the tire and the tunnel floor.

Table 1 - Tested configurations

\begin{tabular}{|c|c|c|c|c|c|}
\hline $\begin{array}{c}\text { Configuration } \\
\text { number }\end{array}$ & $\mathbf{1}$ & $\mathbf{2}$ & $\mathbf{3}$ & $\mathbf{4}$ & $\mathbf{7}$ \\
\hline Ride Height & $\begin{array}{c}\text { Nominal } \\
(65 \mathrm{~mm})\end{array}$ & $\begin{array}{c}\text { Low } \\
(50 \mathrm{~mm})\end{array}$ & $\begin{array}{c}\text { High } \\
(80 \mathrm{~mm})\end{array}$ & Nominal & Nominal \\
\hline $\begin{array}{c}\text { Underfloor } \\
\text { roughness }\end{array}$ & No & No & No & Yes & No \\
\hline Wheels & Yes & Yes & Yes & Yes & No \\
\hline
\end{tabular}
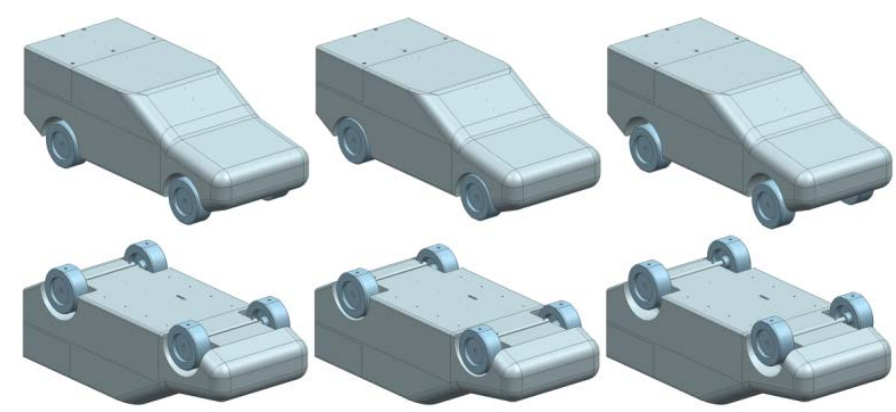

Figure 2 - Ride height variations
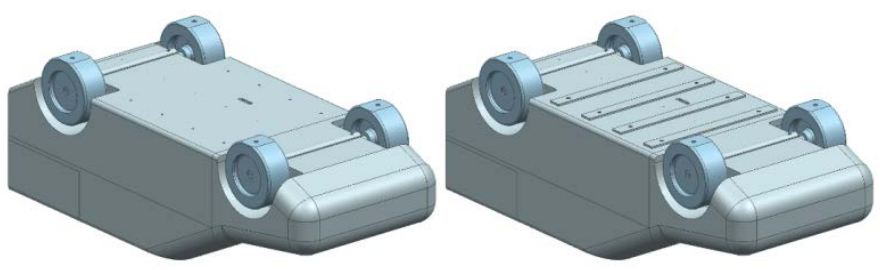

Figure 3 - Underfloor roughness strips

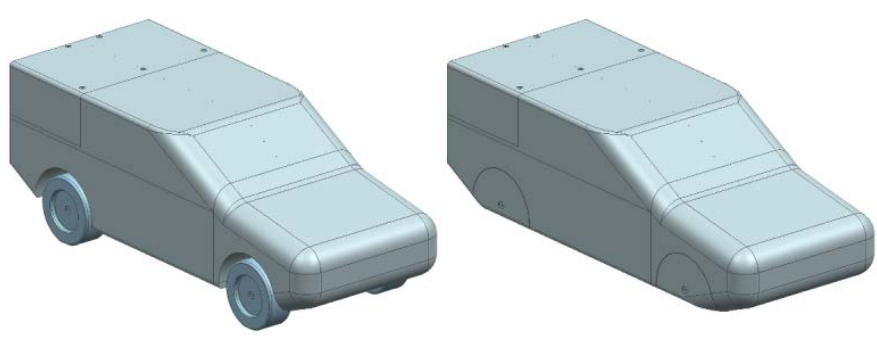

Figure 4 - With and without wheels configurations

As the focus here was to consider the base pressure it was considered useful to make a comparison of the boundary layer development along the model compared to that at full scale. Velocity profiles were recorded using a hotwire system on the model and two full scale SUV models. Data were taken at a range of positions over the length and span of the model and vehicles. The results show a similar form factor validating continued use of the model.

\section{Wind Tunnel}

All testing was conducted in the Loughborough University model scale wind tunnel. The layout is shown in Figure 5. Automotive models up to approximately $1 / 4$ scale can be tested at approximately $5 \%$ blockage. A $140 \mathrm{~kW}$ fan is capable of producing a flow velocity of up to $45 \mathrm{~m} / \mathrm{s}$ in the 1.92 by $1.32 \mathrm{~m}$ working section. The contraction ratio is 7.3:1 and flow conditioning turbulence screens limit freestream turbulence intensity to $0.2 \%$. In the work reported here all testing was conducted at $40 \mathrm{~m} / \mathrm{s}$, giving a Reynolds's number of $2.85 \times 10^{6}$ based on model length. The tunnel is operated in a fixed floor mode with a boundary layer displacement thickness at the model center of $9.4 \mathrm{~mm}$ [5].

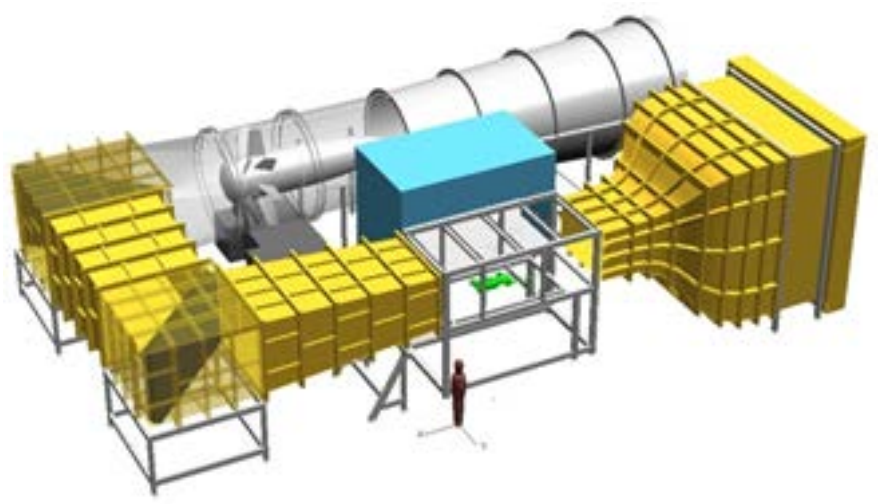

Figure 5 - Loughborough University wind tunnel

\section{Balance Measurements}

Models are mounted onto the six component underfloor balance via four $8 \mathrm{~mm}$ supporting pins that pass through $10 \mathrm{~mm}$ holes in the tunnel floor into holes in the underside of the model wheels, leaving a small clearance under the wheels that have a flattened bottom to represent the contact patch. Adjustment of the supporting pins ensures a consistent setup. The balance and central disk in the tunnel floor are connected to a yaw drive to facilitate automated yaw sweeps. Balance data were sampled for thirty seconds at each yaw position after a ten second settling time. Further details of the balance accuracy can be found in Johl [5], along with further information on the wind tunnel itself. Yaw sweeps were conducted from -20 to $+20^{\circ}$. 


\section{Surface Pressure Measurements}

The base and rear diffuser of the model were populated with a grid of pressure tappings as shown in in Figure 6. The tappings are limited to one half of the model to allow for greater resolution. The pressure measurements were made using two 64 channel miniature pressure scanners located inside the model and accurate to $\pm 0.15 \mathrm{~mm} \mathrm{H}_{2} \mathrm{O}$. Samples were triggered by an externally supplied $260 \mathrm{~Hz}$ signal generator and samples were taken for 31 seconds for each model configuration. Data are recorded against tunnel static pressure.

Pressure coefficients for the model surface have been calculated using the free-stream dynamic pressure (recorded at the start of the working section).All results have been blockage corrected using the MIRA blockage correction (based on continuity), Equation 1.

$\mathrm{C}_{\mathrm{P}_{\text {corr }}}=1-\left(\frac{1-\mathrm{C}_{\mathrm{P}}}{\left(1-\frac{\mathrm{TA}}{\mathrm{A}}\right)^{-2}}\right)$

TA - Tunnel Area

A - Model Frontal Area

$\mathrm{C}_{\mathrm{P}}$ - Recorded Surface Pressure Coefficient

$\mathrm{C}_{\text {Pcorr }}-$ Continuity Corrected Pressure Coefficient

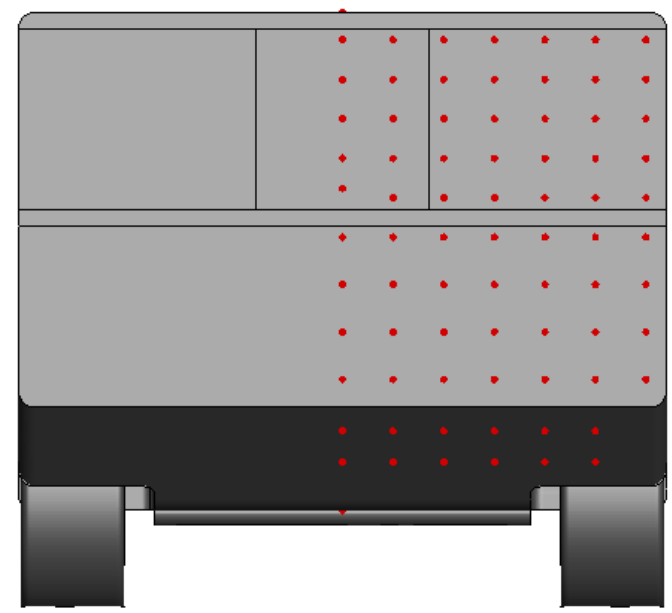

Figure 6 - Surface pressure measurement locations

Mean pressure coefficients, $\overline{C_{p}}$, for the base and diffuser are presented separately and additionally the base + diffuser $\overline{C_{p}}$, which is based on the relative rearwards facing area of each constituent part, is calculated.

\section{Particle Image Velocimetry (PIV)}

Two-dimensional planar PIV measurements have been conducted in a number of planes in the vehicle wake region, as represented in Figure 7. The two horizontal planes are at half upper base height (blue) and half lower base height (red). The vertical planes are the vehicle centerline (green), the wheel centerline (purple) and a location midway between these planes (yellow).

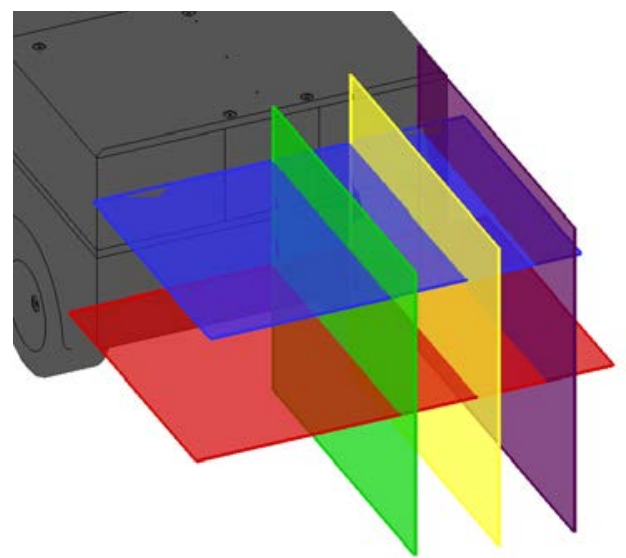

Figure 7 - PIV measurement planes

Dual-frame images were acquired with a 4 mega pixel, 14bit camera equipped with a $50 \mathrm{~mm}$ lens in combination with a frequency doubled Nd:YAG Litron LASER with 200mJ pulse energy, operating using the LaVision DaVis software. The image area was $\sim 400 \mathrm{X} 400 \mathrm{~mm}$ giving a spatial resolution of $0.2 \mathrm{~mm} /$ pixel. 1000 image pairs were captured for each plane at a recording frequency of $7.26 \mathrm{~Hz}$.

\section{CFD Methodology}

Computational validation of the wind tunnel tests was performed using Exa PowerFLOW, a commercial code that utilizes the Lattice Boltzmann Method (LBM). This method is a special discretization of the Boltzmann equation in space, time and velocity. It simulates the flow and collision processes of particles within a Newtonian fluid [6]. The turbulence model employed in the code is similar to Very-Large Eddy Simulation (VLES) with a $\mathrm{k}-\varepsilon$ RNG model acting as a subgrid scale model. The LBM solves the transient, turbulent flow of air around the SUV and the solution is averaged in time to give the mean flow solution.

Spatial discretization in PowerFLOW generates what is known as a lattice, containing 'voxels' (cuboidal volume cells) and 'surfels' (surface cells generated as a voxel intersect a surface). The lattice used for this study was generated using a best practices template currently employed by a leading company in the automotive industry with automated voxel sizes (minimum $0.5 \mathrm{~mm}$ ) and refinement regions based upon model dimensions and geometric features. The smallest voxel size generated was $10^{-3}$ percent of the model length. A cross-section of the lattice shows the locations of refinement around the vehicle, figure 1 . The total number of voxels in the fluid domain is approximately 70 million with $\mathrm{y}^{+}$values in the range of $15 \leq \mathrm{y}^{+} \leq 100$ over the surfaces of the SUV, hence wall functions are applied for the entire surface. 


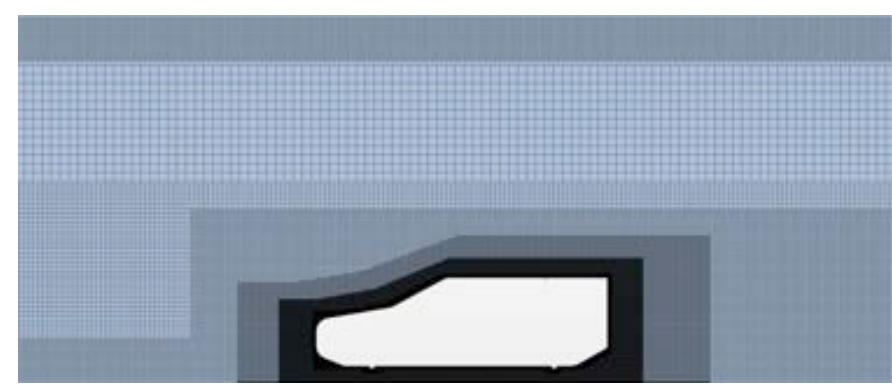

Figure 8 - Voxel refinement around the SUV

A velocity inlet and pressure outlet are used, with slip walls defined until the start of boundary layer growth, (4.5m upstream of the origin) at which point no-slip wall conditions are applied to all tunnel and SUV surfaces. To replicate the experimental conditions, values for free stream velocity and turbulence intensity are set to $40 \mathrm{~m} / \mathrm{s}$ and $0.15 \%$ respectively and Reynolds number maintained at $2.85 \times 10^{6}$ based on model length. All pressure and force results are corrected for blockage effects in the same way as the experimental results.

\section{Experimental Results and Discussion}

\section{Baseline Model}

As the baseline model, i.e. the nominal ride height $(65 \mathrm{~mm})$, smooth floor, with wheels, is used as a basis for comparison for all other models the wake characteristics of this configuration are discussed first. Coefficients of drag, total lift and front and rear lift are presented in Figure 9. The drag and lift results are broadly typical of an SUV type vehicle but there is negative lift at the rear axle over quite a wide range of yaw angles that would not be expected on a production vehicle. This negative lift is attributed to the short diffuser that is an artifact of the method of developing the model, where the large departure angle seen on this vehicle type results in a short upsweep at the rear. Combined with the smooth underbody this diffusing section is then responsible for the rear lift characteristic.

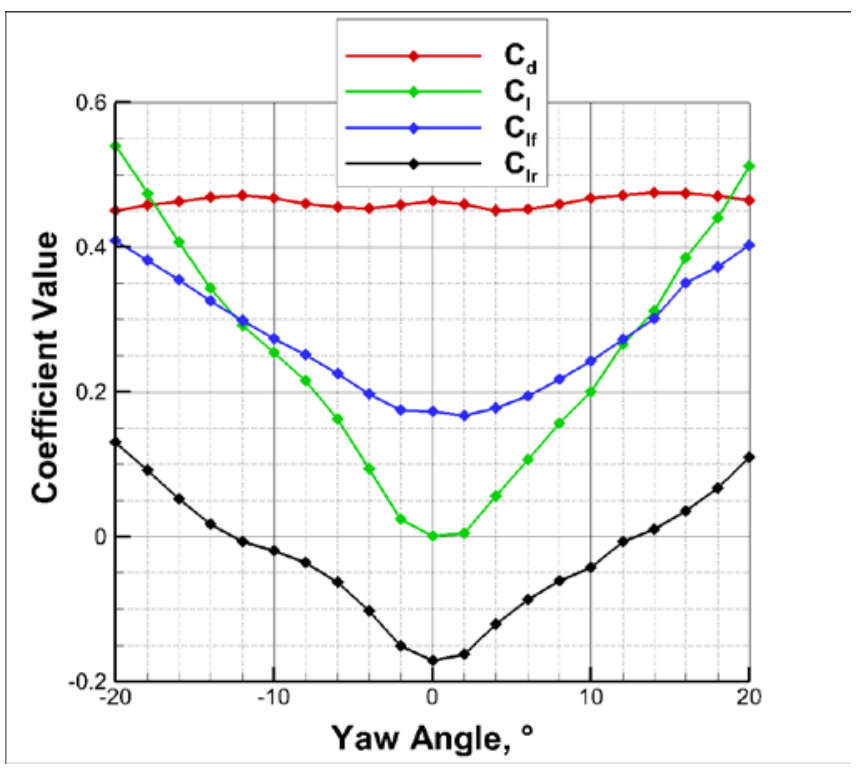

Figure 9 - Drag and lift coefficients for baseline model
The plots shown in Figure 10 for the baseline configuration show the base pressure distribution and velocity streamlines of the flow field on the five measurement planes in the vehicle wake. The pressure distribution on the upper base area shows iso-lines of constant pressure coefficient that are approximately horizontal. This suggests that the pressure recovery is mainly due to flow from above the model as opposed to from the vehicle sides. The effect of the wheel wakes is also clearly evident in the bottom outer quarter on both the diffuser and the lower base where there is increased suction compared to the rest of the base. The mid plane upper glasshouse PIV data show a symmetrical velocity field with limited inward flow from the vehicle sides. The structures on the lower base (mid plane lower glasshouse) are less symmetrical and the influence of the wheel wakes is very evident and suggests a highly three dimensional structure.

The vertical planes of PIV might all be characterised by the expected upper and lower recirculations but are in practice quite different at the three lateral locations. On the vehicle centreline the flow exiting the diffuser suggests that it is fully attached. This is unexpected for a $30^{\circ}$ diffuser and is certainly due to the influence of the wheels. Otherwise at this plane the upper and lower vortex structures are as reported in the literature for the square-back geometry [7]. However at mid vehicle width the upper vortex structure is not clearly present at all and is replaced with an extended shear layer. Without the upper vortex the lower recirculation dominates the wake and is more closely aligned with the model base as the upper section is no longer distorted by the upper vortex. This influences the mean wall velocities and likely the base pressures. At the wheel centreline the upper vortex is again present and the lower is now much reduced in strength as the flow in this region is restricted by the presence of the wheels. The flow exiting the diffuser is not attached in either of the off centreline velocity fields.

Though the images are not included in this paper the small upper structure seen in the time averaged velocity field is not observed in the instantaneous flow fields. In the instantaneous fields the region around the upper shear layer is typically made up of many smaller vortices that are convected downstream in a highly unsteady region. The recirculation shown is merely the agglomeration of many of these smaller vortices. 


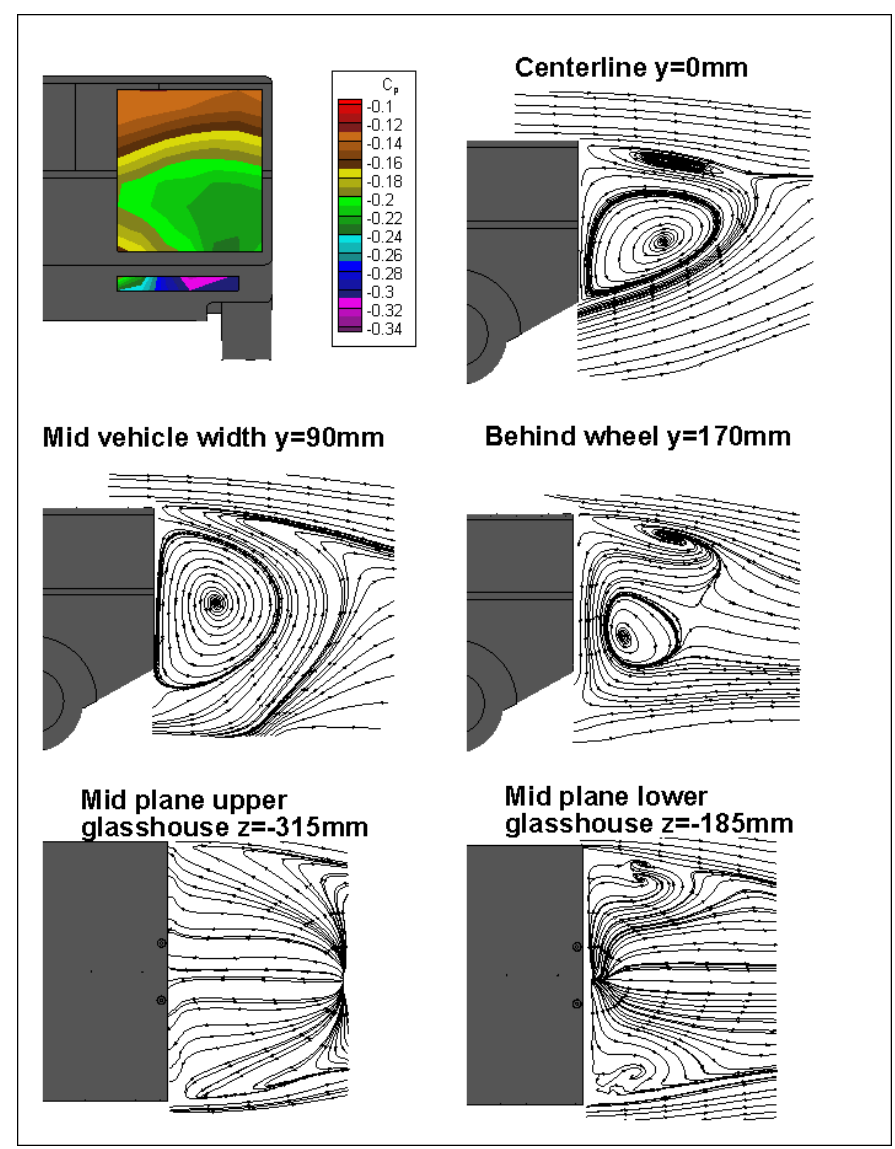

Figure 10 - Nominal ride height, smooth floor, with wheels, pressure and PIV results

\section{Effect of Ride Height}

The model was tested at three ride heights: nominal (65mm), low (50mm) and high $(80 \mathrm{~mm})$. The nominal case being the data for the baseline reported above. The changes were implemented by moving the model body relative to the wheels so that the small clearance under the wheels, required to prevent grounding of the balance in the fixed floor arrangement, was maintained. This approach results in a small increase or reduction in frontal area so the relevant reference area is used in the calculation of the coefficients in each case. For the high ride height case the increase in frontal area is $1.6 \%$ there is a decrease of $1.4 \%$ for the low ride height case, both relative to the nominal ride height model. It is worth noting that with the fixed floor arrangement the effect of the boundary layer is likely to be significant but that the basic mechanisms are unlikely to be changed.

The drag characteristics for the three configurations, Figure 11, show, as expected, an increased $C_{d}$ for the high ride height case of $6.7 \%$ and a reduced $\mathrm{C}_{\mathrm{d}}$ for the low ride height case (4.3\%) compared to the baseline.

Page 5 of 10

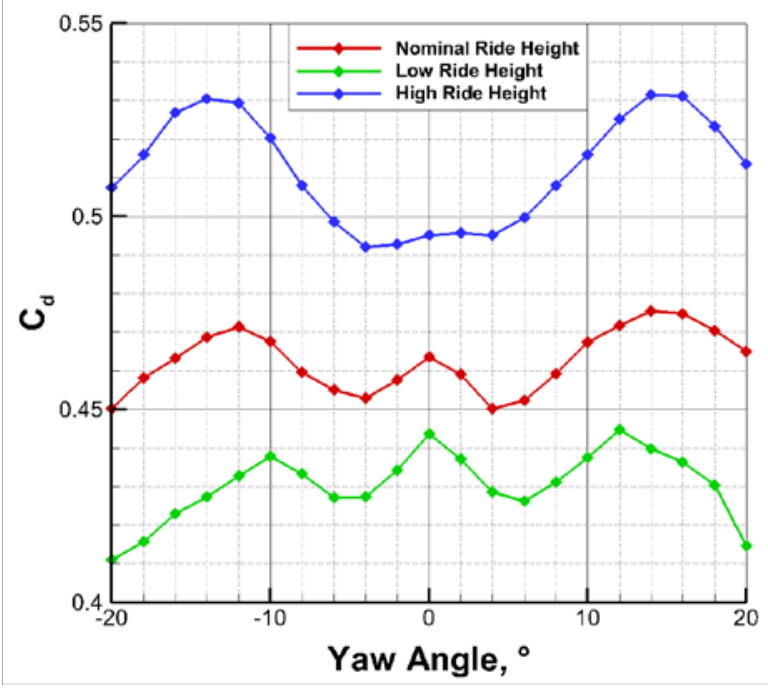

Figure 11 - Drag coefficients for ride height variations

Increasing the ride height from nominal to high has little effect on the total lift, Figure 12, as the ground clearance is sufficient in both cases for there to be limited ground effect. However, at the lowest ride height there is a significant decrease in lift that is attributed to different flow behaviour in the underbody compared to the nominal condition.

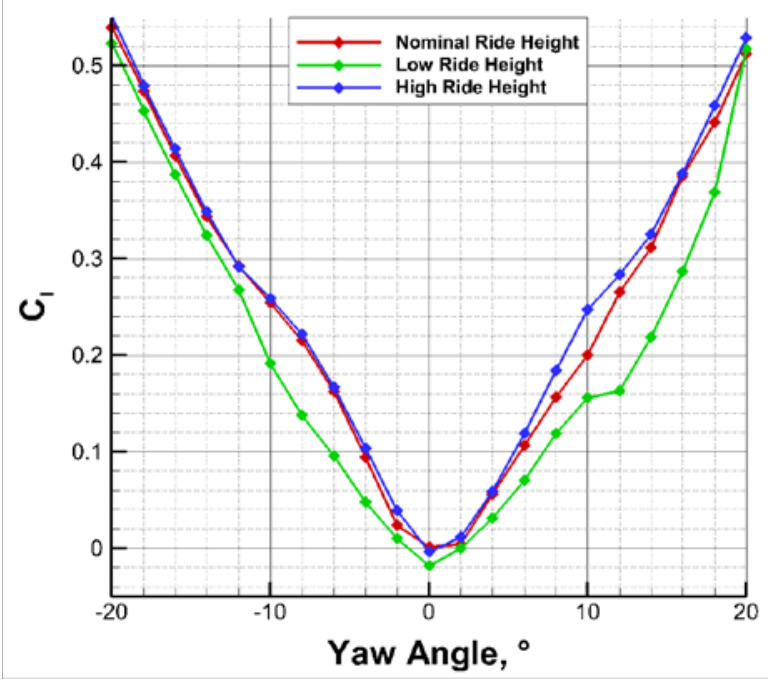

Figure 12 - Lift coefficients for ride height variations

The base pressure plots in Figure 13 and Figure 14 for the different ride heights show a general trend towards lower base pressure with increasing ride height. This is in part due to the increased model area producing a larger and lower pressure wake. The PIV data in the vertical plane confirms this, showing a shorter distance behind the model before the recirculations close for the lower ride height configuration. This is most evident for the mid-model width PIV plane results. Further evidence of this is also show by the location of the rear stagnation point in the upper glasshouse horizontal PIV results, with this point moving further downstream with increasing 
ride height. The effect of the wheel wakes is quite clear in the pressures with the extent of the associated lower pressure region increasing at the higher ground clearances. There is also a reduction in pressure on the diffuser in the wheel wake area.

The mean pressure data ( $\overline{C_{p}}$ ) for the base and diffuser are shown in Table 2 in addition the $\overline{C_{p}}$ for the base and diffuser combined is shown. The increase in pressure on both the base and the diffuser at low ride height compared to the nominal is significant and clearly is the main contributor to the reduction in drag. However, there is much less of a difference in the pressure between the nominal and high ride height cases with the largest effect arising in the diffuser. Overall it is clear that the base pressure is not responsible for the increase in drag and that it is more likely due to the increased exposure of the wheels and higher fore-body drag as the position of the front stagnation changes.

Table $2-\overline{C_{p}}$ with varying ride height

\begin{tabular}{|l|l|l|l|}
\hline Configuration & Low & Nominal & High \\
\hline Diffuser $\overline{\boldsymbol{C}_{\boldsymbol{p}}}$ & -0.257 & -0.285 & -0.297 \\
\hline Base $\overline{\boldsymbol{C}_{\boldsymbol{p}}}$ & -0.151 & -0.161 & -0.162 \\
\hline $\begin{array}{l}\text { Base and diffuser } \overline{\boldsymbol{C}_{\boldsymbol{p}}} \\
\text { combined }\end{array}$ & -0.182 & -0.195 & -0.198 \\
\hline Total drag & 0.444 & 0.464 & 0.495 \\
\hline
\end{tabular}
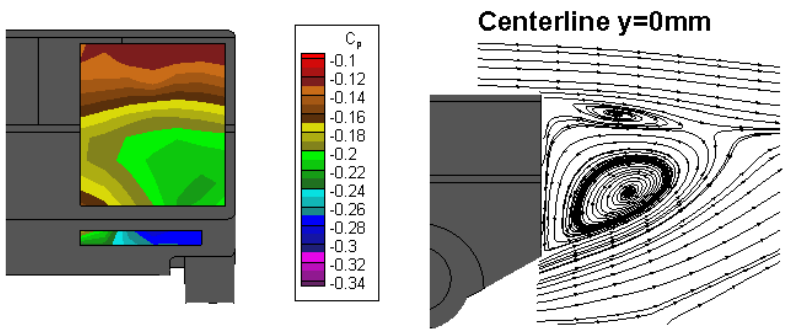

Mid vehicle width $y=90 \mathrm{~mm}$

Behind wheel $y=170 \mathrm{~mm}$
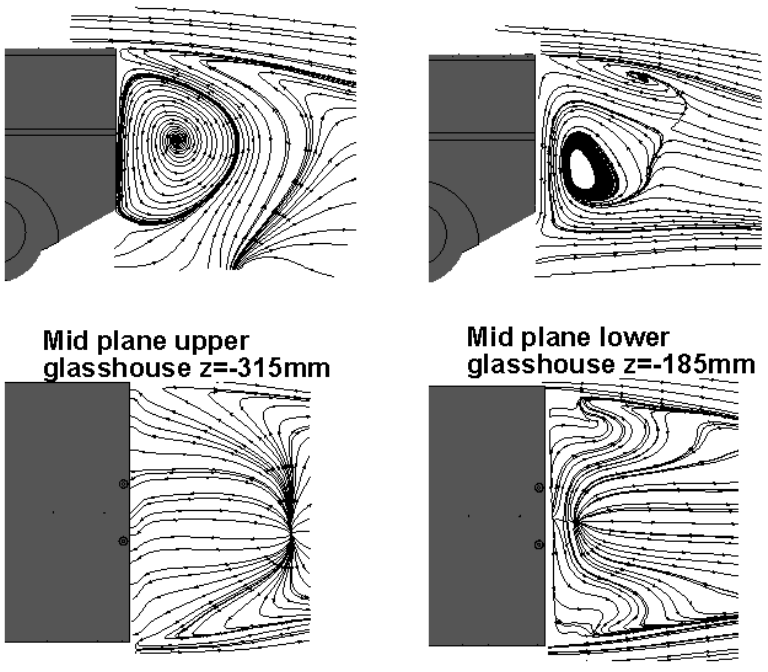

Figure 13 - Low ride height, smooth floor, with wheels, pressure and PIV results

Comparing the PIV for the three cases more generally the essential features do not appear to be altered greatly, particularly when the Page 6 of 10 nominal and low ride heights are compared. In that case there is some strengthening of the lower recirculation in all three planes and also some changes to the velocity gradients that may feed in to the subtle changes in base pressure. At the highest ride height the mid-vehicle width plane shows a more typical upper recirculation rather than the sheared flow seen for the other configurations. Also for this plane the streamlines in the lower recirculation suggest much stronger crossflow that is also evident in the other two planes.

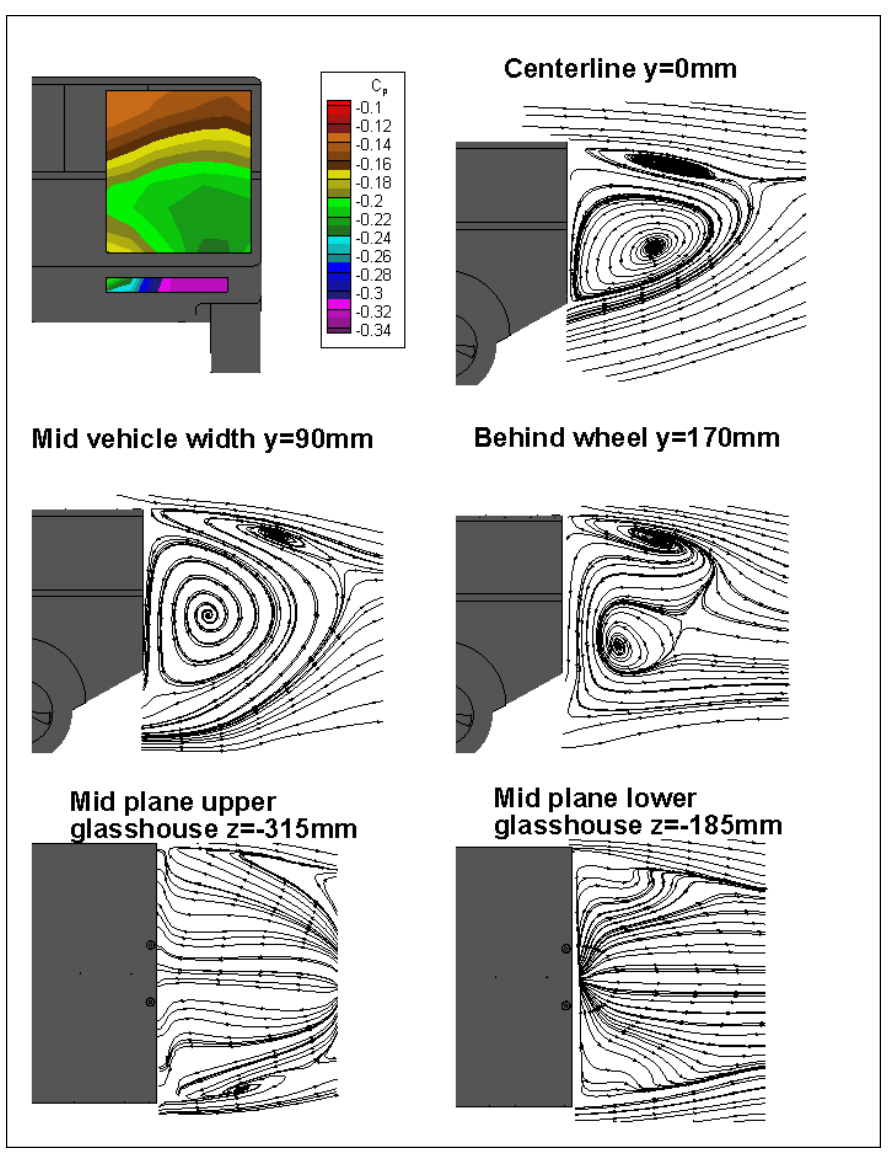

Figure 14 - High ride height, smooth floor, with wheels, pressure and PIV results

\section{Effect of Underfloor Roughness}

Comparing the standard smooth floor with a rough underbody, Figure 15 shows only small changes in the overall drag characteristic particularly at the lower yaw angles. In practice the roughness strips are of similar size as the wheel axles, so may not contribute a great deal of additional disturbance to the underbody flow. At higher yaw $\left(>5^{\circ}\right)$ there is a larger increase in drag for the rough floor case. That is because at higher yaw angles the roughness strips are not hidden behind the axles, thus have a greater effect. The lift is essentially unchanged by the addition of roughness and hence the results are not included. 


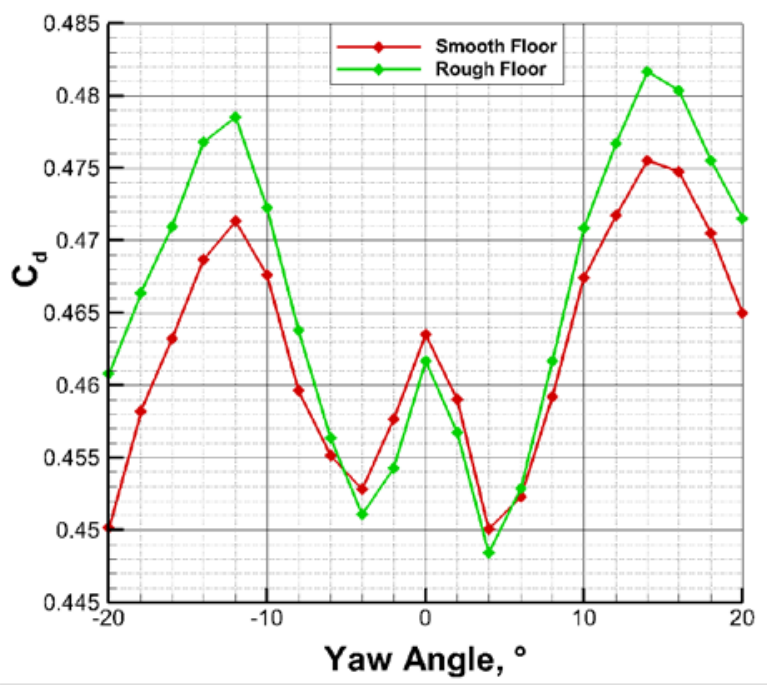

Figure 15 - Drag coefficients for underfloor roughness variations

The base pressure and PIV data, Figure 16, shows a large degree of similarity to that of the baseline model further confirming the hypothesis that at zero yaw the roughness strips have only a small effect. There is some change to the shear layer exiting the diffuser on the centreline case and some small changes to the interaction between the upper and lower vortex structure behind the wheel. These small changes are reflected in the area weighted pressure data ( $\overline{C_{p}}$ ), Table 3 , that shows a small increase in base and diffuser pressure for the rough floor model that accounts for the small decrease in drag.

Table $3-\overline{C_{p}}$ with smooth and rough floor

\begin{tabular}{|l|l|l|}
\hline Configuration & Smooth floor & Rough floor \\
\hline Diffuser $\overline{\boldsymbol{C}_{\boldsymbol{p}}}$ & -0.285 & -0.278 \\
\hline Base $\overline{\boldsymbol{C}_{\boldsymbol{p}}}$ & -0.161 & -0.157 \\
\hline $\begin{array}{l}\text { Base and diffuser } \overline{\boldsymbol{C}_{\boldsymbol{p}}} \\
\text { combined }\end{array}$ & -0.195 & -0.191 \\
\hline Total drag & 0.464 & 0.462 \\
\hline
\end{tabular}

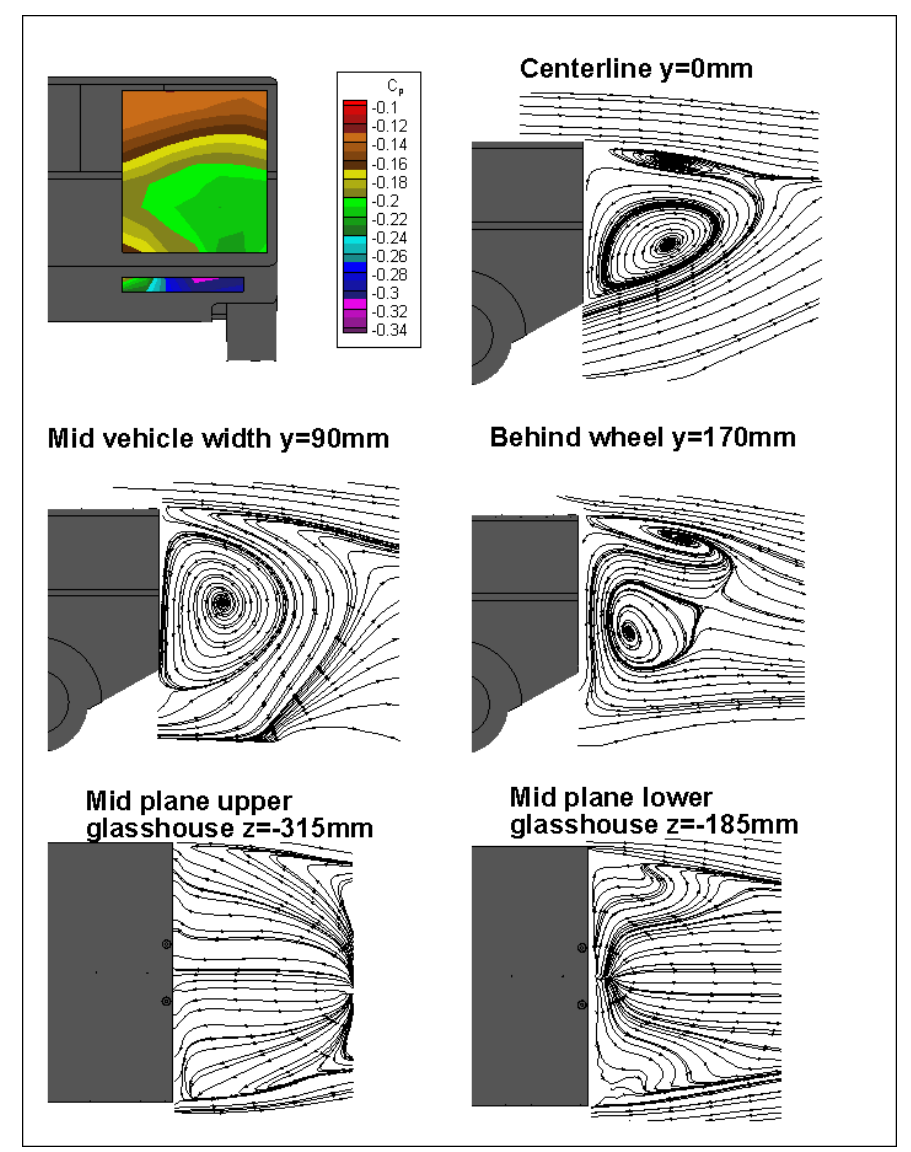

Figure 16 - Nominal ride height, rough floor, with wheels, pressure and PIV results

\section{SUV model without Wheels}

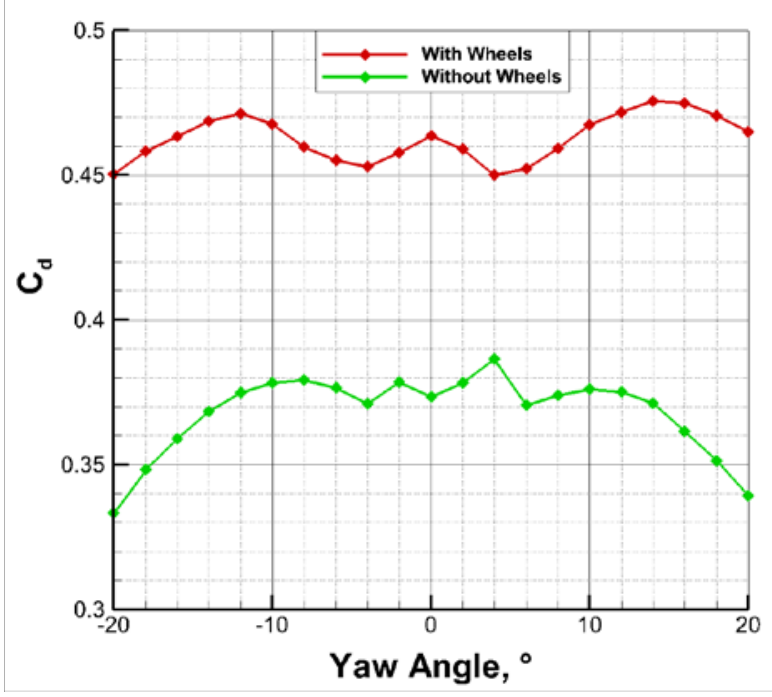

Figure 17 - Drag coefficients for with and without wheels models 
Many of the published reference bodies and simplified geometries are either designed without wheels, to avoid the unnecessary complication, or have the option of a test case without wheels. For the SUV geometry here the without wheels case uses a filler to entirely fill the wheel arch in place of the wheels Figure 4. The balance data, Figure 17 and Figure 18, show similar profile curves but a drag coefficient reduction of approximately 0.1 and a lift offset of around -0.5. This suggests some quite fundamental differences to the flow-field and also illustrates the major contribution of the wheels, which account for only an extra 5\% in model frontal area, to the drag.

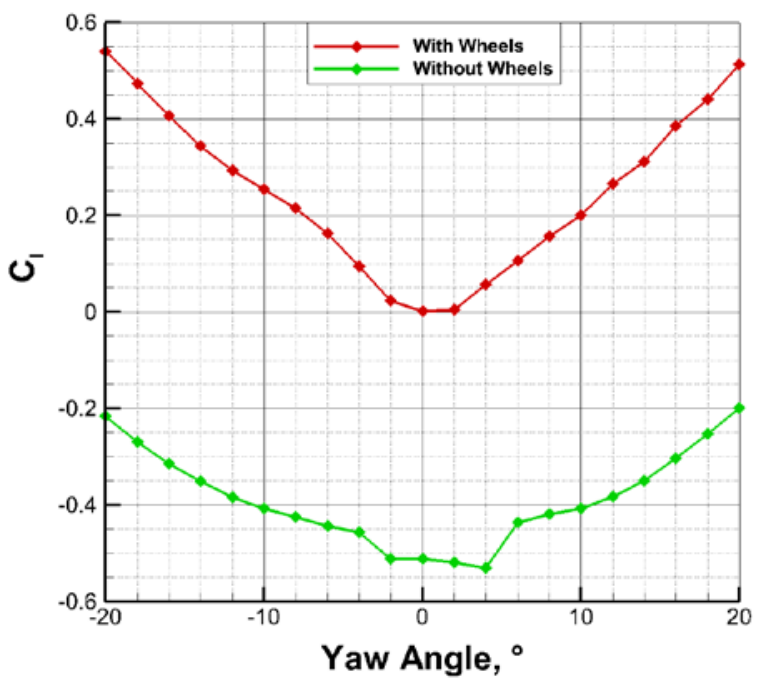

Figure 18 - Lift coefficients for with and without wheels models

The base pressure distribution and wake flow fields, Figure 19, show very different behaviour for the model with wheels and without. The usual large lower vortex and small upper vortex are present but apart from this the results are quite different. On the centreline the flow exiting the diffuser without wheels is no longer attached as it is when the wheels are present. However, it is fully attached outboard of the centreline at the mid vehicle width because the up-wash generated at the diffuser edges is no longer blocked by the presence of wheels. This explains the differences in the shapes of the drag and lift curves at low yaw angles, as it is likely the attached flow observed for the with wheels case is quite sensitive to the onset condition and hence the yaw angle. Generally the wake closes more quickly for the case without wheels producing a smaller wake, changes to the wall velocities and higher base pressure.

The location of the lowest pressure is in the middle of the base for this configuration is as expected of a simple bluff body. The higher pressure to the sides of this area is due to air being drawn from the model sides, as shown on the lower base horizontal PIV flow field. This low pressure zone is also shown to draw down air from above the vehicle on the centreline, hence the higher pressure on the upper base on the centreline than the edges, contrary to the with wheels cases.

Changes to the structure of the wake are particularly evident on the lower base where the influence of the wheel wakes is greatest. For the cases with wheels the flow is shown to be travelling outwards from the vehicle centreline towards the low pressure in the wheel wakes. Without the wheels the flow migrates towards the centreline as this is now the lowest pressure area.

The mean pressure data $\left(\overline{C_{p}}\right)$, Table 4 , shows, as expected, a significant increase in $\overline{C_{p}}$ on both the base and diffuser when the wheels are removed. This explains a significant proportion of the reduced overall drag.

Table $4-\overline{C_{p}}$ with and without wheels

\begin{tabular}{|l|l|l|}
\hline Configuration & With wheels & Without wheels \\
\hline Diffuser $\overline{\boldsymbol{C}_{\boldsymbol{p}}}$ & -0.285 & -0.271 \\
\hline Base $\overline{\boldsymbol{C}_{\boldsymbol{p}}}$ & -0.161 & -0.126 \\
\hline $\begin{array}{l}\text { Base and diffuser } \overline{\boldsymbol{C}_{\boldsymbol{p}}} \\
\text { combined }\end{array}$ & -0.195 & -0.152 \\
\hline Total drag & 0.464 & 0.373 \\
\hline
\end{tabular}

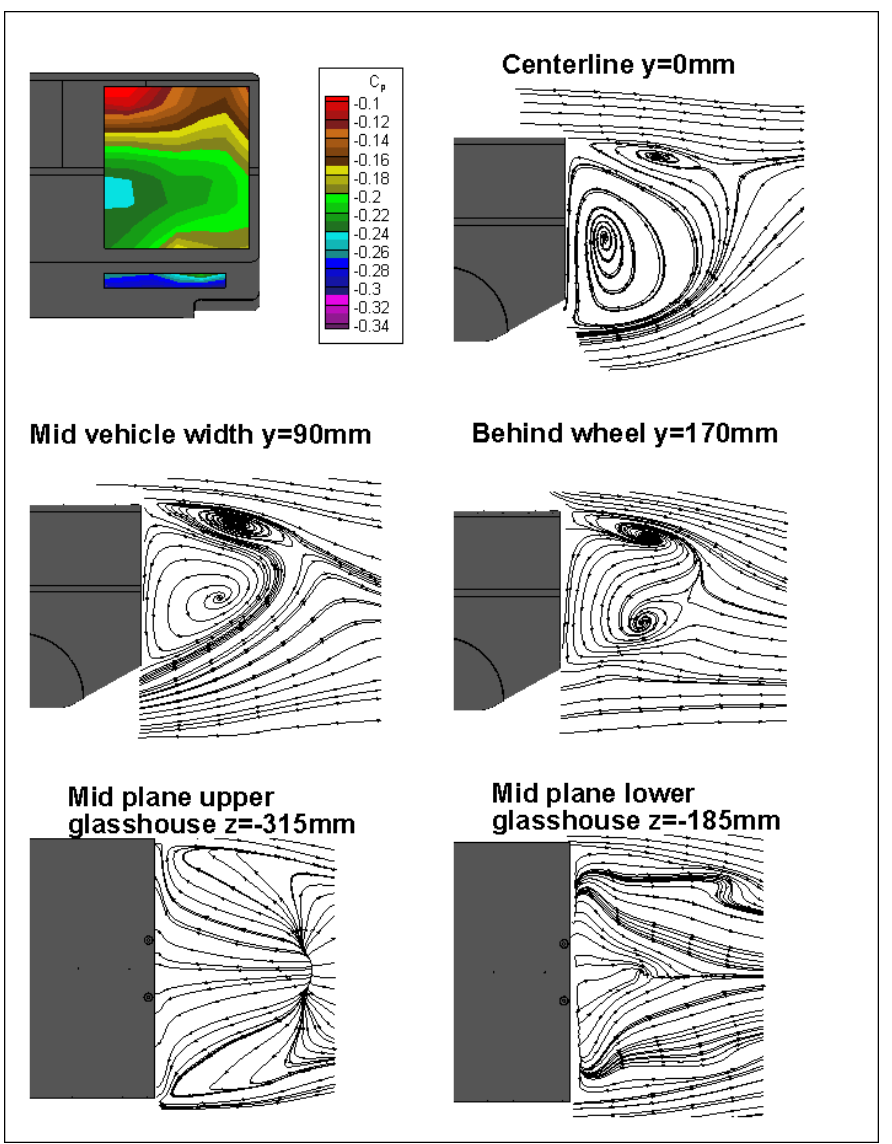

Figure 19 - Nominal ride height, smooth floor, without wheels, pressure and PIV results

\section{CFD Comparison}

This section compares the experimental data presented thus far with numerical work completed as part of a complimentary project at Loughborough University [8]. The comparison is only presented for the baseline configuration; with wheels a smooth floor and at the nominal ride height.

A basic comparison, Figure 20, shows the base pressure values to be reasonably well predicted, with difference of the order 0.05 in $C_{p}$. 
The shape of the pressure distribution is also similar for the experiment and simulation. The integrated difference in base pressure for the simulation compared to the experiment correlates with a reduction in drag coefficient from 0.464 to 0.434 .

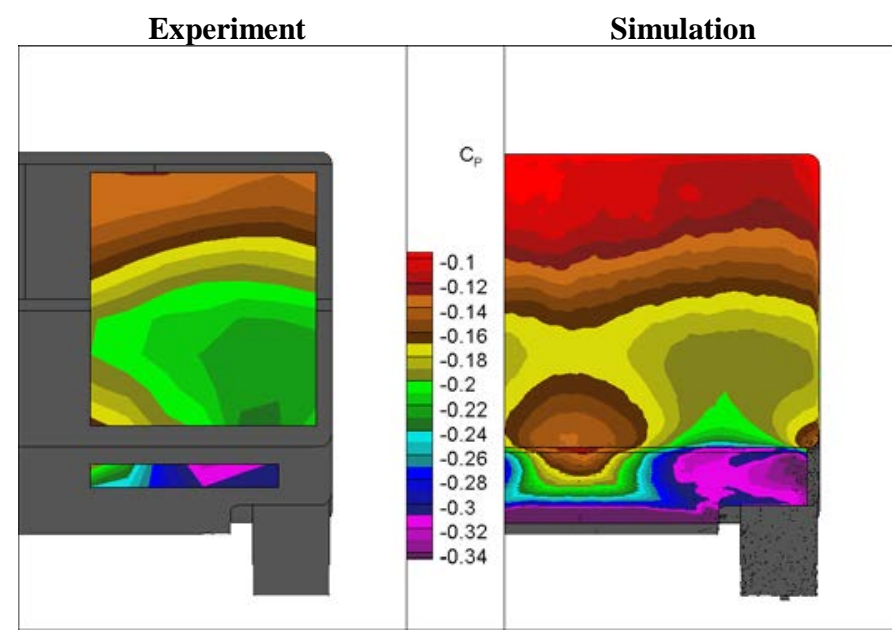

Figure 20 - Base pressure comparison between experiment and simulation in nominal ride height, smooth floor, with wheels configuration

There is a good ability shown by the simulation to predict the low pressure zone in the wheel wake. However, the simulation appears to over predict the effect of the air from the side increasing the pressure to this side of the wheel wake. The simulation also captures the region of increased pressure at the top of the diffuser on the model centerline.

\section{Summary/Conclusions}

1. A model was created to be representative of modern and future SUV's.

2. The generic SUV model was tested in a number of ride height, underfloor roughness and with/without wheel configurations.

3. Balance, pressure and wake PIV data were taken and are presented for all configurations along with the model geometry. These data will be available as a validation set for CFD predictions of base pressure. This can be accessed from the Loughborough University institutional repository [1].

4. Changes between the wake structure and the resultant base pressure were shown. Particularly the rearwards extent of the main bulk motions and the level of negative pressure on the base region. Further investigation of the effect of wall velocities on base pressure is needed.

5. The PIV results show that when the wheels are present the flow is attached at exit from the $30^{\circ}$ diffuser on the vehicle centerline. This demonstrates that the diffuser operates quite differently with and without wheels and may be a useful observation for future vehicle optimization.

6. Changing ride height was shown to influence the size of the main bulk flow within the wake and therefore the pressure on the base.

7. Underfloor roughness strips were shown to have only a small effect on the overall drag but that the change was attributable to changes in the base pressure.

\section{References}

1. Institutional repository https://dspace.lboro.ac.uk/dspace-jspui/

2. Ahmed, S., Ramm, G., and Faltin, G., "Some Salient Features Of The Time-Averaged Ground Vehicle Wake," SAE Technical Paper 840300, 1984, doi: $10.4271 / 840300$

3. Le Good, G. and Garry, K., "On the Use of Reference Models in Automotive Aerodynamics," SAE Technical Paper 2004-011308, 2004, doi:10.4271/2004-01-1308

4. Newnham, P., Passmore, M.A. and Baxendale, A., "On the optimisation of road vehicle leading edge radius in varying levels of freestream turbulence,” SAE Technical Paper 200601-1029, 2006, doi:10.4271/2006-01-1029

5. Johl, G., Passmore, M.A. and Render, P.M., "Design methodology and performance of an indraft wind tunnel” The aeronautical journal, 108(1087): 465-473, 2004

6. $\quad$ Lietz, R., Mallick, S., Kandasamy, S., and Chen, H., "Exterior Airflow Simulations Using a Lattice Boltzmann Approach," SAE Technical Paper 2002-01-0596, 2002, doi:10.4271/2002$\underline{01-0596}$

7. Ahmed, S.R., “An Experimental Study of the Wake Structures of Typical Automobile Shapes" Journal of Wind Engineering and Industrial Aerodynamics, 9: 49-62, 1980

8. Forbes, D.C., Page, G.J., Passmore, M.A., Gaylard, A.P., "Computational study of wake structure and base pressure on a generic SUV model," International Vehicle Aerodynamics Conference, 67-79, 2014

\section{Contact Information}

Martin Passmore: m.a.passmore@lboro.ac.uk

The full data sets can be accessed from the Loughborough University institutional repository. Please see reference 1 .

The data includes details of the model geometry, model setup in the wind tunnel the time averaged and RMS PIV data, the surface pressure data and the force and moment data.

\section{Acknowledgments}

The authors would like to thank the technical staff at Loughborough University, Dave Cooper and Rob Hunter, for their excellent work in model preparation and tunnel maintenance.

\section{Definitions/Abbreviations}

PIV

Particle image velocimetry

$\mathbf{C}_{\mathbf{D}}$

$\mathrm{C}_{\mathbf{P}}$

Drag coefficient

$\mathbf{C}_{\mathrm{L}}$

Pressure coefficient

$\mathrm{C}_{\mathrm{LF}}$

Lift coefficient

$\mathrm{C}_{\text {LR }}$

Rear lift coefficient 
Page 10 of 10 